\title{
Effect of Implementing Nursing Educational Protocol on Reducing Deep Venous Thrombosis among Orthopedic Surgery Patients
}

\author{
Mona Rabea Mohamed Ahmed ${ }^{1}$ Shalabia El-Sayed AboZead ${ }^{2}$, Ahmed Omar Yousef ${ }^{3}$, \\ Sahar Hamza Taha ${ }^{4}$
}

1. B.Sc. Nursing, Faculty of Nursing, Minia University.

2. Professor of Medical- Surgical Nursing, Faculty of Nursing, Assuit University.

3. Professor of Orthopedic Surgery, Faculty of Medicine, Minia University.

4. Lecturer of Medical Surgical Nursing, Faculty of Nursing, Minia University.

Corresponding author: adamwaly11@gmail.com

\begin{abstract}
:
Background: Deep venous thrombosis is a most prevalent problem for orthopedic surgery patients. Nurses have been shown in the literature to play a significant role in reducing the incidence of deep venous thrombosis. Range of motion exercises increase blood flow to the lower extremities, and early mobilization has been shown to improve outcomes. Nurses are crucial in integrating these activities into their patients' regular care plans. Future research should concentrate on efforts to enhance nursing compliance with this exercise program .Aim of the study: To evaluate the effect of implementing nursing educational protocol on reducing deep vein thrombosis among orthopedic surgery patients. Research design: Quasi experimental research design was utilized to conduct this study (study and control group). Subjects: A purposive sample of 72 patients (36 patients for each group study and control). Setting: This study was carried out in orthopedic department and orthopedic outpatient clinic at Minia University Hospital. Tools of data collection: Demographic and medical sheet, the Autar DVT risk assessment scale and Deep vein thrombosis evaluation sheet. Results: There was a statistical significant difference between study and control groups regarding the occurrence of DVT especially Left limb DVT p.value $\left(0.027^{*}\right)$ after application of the nursing educational protocol. There were highly statistically significant differences between study and control groups regarding signs and symptoms of DVT in calf muscle regarding redness, swelling and absence of signs of DVT with p.value $(0.012 *, 0.001 * *$, and $0.002 * *)$ respectively. While in thigh muscle presence of swelling show a statistical significant difference with p.value $\left(0.005^{* *}\right)$ between the two groups. There was a statistical significant difference between study and control groups regarding the occurrence of DVT especially Left limb DVT p.value $\left(0.027^{*}\right)$ after application of the nursing educational protocol. Conclusion: Application of educational protocol was effective in reducing DVT among orthopedic surgery patients. Recommendations: Applying elastic stocking, exercising and range of motion had a positive effect on reducing incidence of DVT among orthopedic surgical patients. The inservice educational training program that illustrates purposes and benefits of applying elastic stocking, exercising and range of motion is very important.
\end{abstract}

Keywords: Educational protocol, Deep venous thrombosis, Nursing, Orthopedic surgery.

\section{Introduction}

Deep vein thrombosis (DVT) is the formation of thrombi in the deep veins, most commonly the large veins of the legs or pelvis. Pulmonary embolism (PE) develops when thrombi dislodge from clots in vein walls and travel through the heart to pulmonary arteries. There is a $50 \%$ chance for patients with untreated proximal DVT to develop symptomatic PE within 3 months. For $25 \%$ of patients, the presenting manifestation of $\mathrm{PE}$ is sudden death Phillippe (2017).

Patients undergoing major orthopedic surgery including total hip replacement, total knee replacement and hip fracture surgery, are at particular risk of developing postoperative venous thromboembolism (VTE) (Francis, 2013).

Orthopedic patients are at higher risk among all patients for DVT and venous thromboembolism (VTE), the incidence of DVT ranges up to $40 \%$ to $60 \%$ in major orthopedic surgeries. Death within one month of diagnosis occurs in approximately $6 \%$ of DVT patients and approximately $12 \%$ of pulmonary embolism patients (Flevas et al., 2018).

Deep vein thrombosis (DVT) is an important cause of morbidity and mortality. The risk of DVT is highest in patients undergoing major orthopedic surgery, particularly hip and knee arthroplasty (Akpinar, Hoşgün, Akan, Ateş, \& Gülhan, 2013).

Major surgery or traumas of the lower extremities trigger the coagulation cascade and the physiologic equilibrium between factors promoting, and retarding coagulation is disturbed resulting in a hypercoagulable state. In these patients, a reduced venous flow and impaired endothelial function further increase the risk of developing deep-vein thrombosis and pulmonary embolism (Dahl, 2013 ).

The most common symptoms of DVT occur at the site of the clot. They include swelling, warmth, pain or tenderness, and redness of the skin. Deep vein thrombosis can also occur with no symptoms (Thompson, 2015).

Patients undergoing major orthopedic surgery should receive thromboprophylactic interventions, including health education, properly implemented basic prevention strategies, physical prevention strategies, and appropriate antithrombotic drugs. Ideally, these interventions should be patient-centered in order to encourage patient participation and adherence $(\mathbf{X u}$, Zhao, \& Chen, 2018).

Nurses caring for patients at risk for DVT need to 
consider their health history, family history, current illness, current medications, and physical assessment data. To effectively advocate for patients and prevent DVT, nurses have a responsibility to assess the patient's risk factors and identify and promptly report abnormal physical assessment findings (D'Alesandro, 2016).

\section{Significance of the study}

DVT is more common in orthopedic patients than in the general population, with DVT rates ranging from $40 \%$ to $60 \%$ in major orthopedic surgeries. In approximately $6 \%$ of DVT patients and approximately $12 \%$ of pulmonary embolism patients, death occurs within one month of diagnosis (Flevas et al., 2018). DVT incidence rates without prophylaxis have been observed to range from $40 \%$ to $60 \%$ (Rahman et al., 2020).

Deep venous thrombosis (DVT) is the primary cause of pulmonary embolism (PE) (Schellack, Modau, \& Schellack, 2016). Pulmonary embolism (PE) is a lifethreatening condition or complication and might be one of the worst nightmares for most surgeons. The embolus that causes the obstruction usually travels through the venous system from a distant site.PE causes symptoms such as dyspnea, chest pain or collapse .Moreover, the clinical severity of PE can vary, ranging from asymptomatic cases to sudden death (Attia, Ali, Al Amory, \& Othman, 2015).

Among people who had a DVT, 50\% will have longterm complications (post thrombotic syndrome) such as extremity pain, venous dilation, edema, pigmentation, and venous ulcers (D'Alesandro, 2016).

From the researcher's review of literatures ,It was found that that majority of orthopedic patients return with deep venous thrombosis which can be reduced through mechanical methods such as range of motion, exercises, and elastic stocking wearing .So, this study will be conducted to reduce deep venous thrombosis among orthopedic surgery patientsby application of nursing educational protocolto those patients.

\section{Aim of the Study:}

The aim of this study was to evaluate the effect of implementing nursing educational protocol on reducing deep vein thrombosis among orthopedic surgery patients.

\section{Research Hypothesis:}

The occurrence of deep venous thrombosis will be reduced among orthopedic surgery patients who are following nursing education protocol.

\section{Research Design:}

Quasi experimental research design was utilized in this study (study and control group).

\section{Subjects:}

A purposive sample of 72 adult patients who admitted to orthopedic unit for lower limb orthopedic surgery. The determination of the sample size based upon the following sample calculation formula Sample size is calculated by using Naing, Winn, \& Rusli , (2006) formula which is computed as $\left(n=z^{2} \times p(1-p) / d^{2}\right)$ (Naing, Winn, \& Rusli Bin, 2006) .
Setting:

The study was conducted at Orthopedic department and Orthopedic out Patient Clinic at Minia University Hospital.

\section{Study Duration:}

The total data collections were collected over a period of ten months starting from November 2019 to August 2020.

\section{Tools of data collection}

Three tools were used for data collection of this study:

Tool I: Patient's Assessment Sheet for DVT

This tool aimed to assess the patients' risk factors for development of DVT and it consisted of three parts:-

- Part 1: Demographic data of the patient: Such as age, sex, educational level, family history, etc.

- Part 2: Patient medical sheet: This part was developed to assess patient medical diagnosis, hospital stay, type of surgery, duration of surgery ,type of anesthesia, type of medications used and patient's hydration state.

- Part 3: the Autar DVT risk assessment scale(Autar, 2003) .

The Autar DVT scale was developed to proactively identify patients at risk for DVT, the Autar DVT scale comprised seven subscales (Age specific group, Mobility, Trauma, Build/Body mass index, Special risk factor, High risk disease, Surgical intervention)

Scoring system of The Autar DVT risk assessment scale

\begin{tabular}{|l|l|}
\hline Risk categories & Score range \\
\hline No risk & $\leq 6$ \\
\hline Low risk & $7-10$ \\
\hline Moderate risk & $11-14$ \\
\hline High risk & $\geq 15$ \\
\hline
\end{tabular}

Tool II: Deep vein thrombosis evaluation sheet This tool was modified by the researcher to assess the development of DVT in order to evaluate the effectiveness of the nursing protocol application. It included three parts:

- Part 1: Deep Vein Thrombosis Manifestations: This part was used to assess DVT manifestations. It assessed the patient in the following five items: calf pain, calf tenderness, calf circumstance, skin color, and warmth of the calf muscle.

- Part 2: Doppler Ultrasound: This test was done to assess the blood supply in the veins of the lower limbs. The Doppler findings indicate the occurrence of DVT. It was done only for patients presented with Deep Vein Thrombosis manifestations.

\section{Educational brochure:}

It was formulated by investigator after extensive literature review and revised by experts. It was included an overview about deep venous thrombosis ,steps of application of elastic stocking, four types of exercise and four types of range of motion for strengthening thigh and calf muscles. Also, instructions regard duration and frequency to ensure that the patients perform these exercises accurately after their hospital discharge (4-6 weeks) and at home. 
Tools validity:

The tools were tested for validity by a jury of five experts in the field of the study and the necessary modifications were done.

\section{Reliability of tools}

To establish reliability, alpha Cronbach's was used to check the stability of the internal consistency of the study instruments which revealed that the tools of the study were reliable as indicated by the value of (0.87).

\section{Pilot Study}

A pilot study was carried out on $10 \%$ (7 patients) of the total sample to test feasibility, objectivity, and applicability of the data collection tools \& scales. Based on results of the pilot study refinement and modifications was done by the researcher. So that the researcher was found more applicable to current study to use a standardized scale as (Autar DVT Risk Assessment Scale) .

\section{Ethical Consideration}

Approval was obtained from the ethical committee in the faculty of Nursing, the director of Orthopedic Surgical Unit. Oral consents were obtained from subjects, and explanation about the study was given to them included the aim of the study. Confidentiality and anonymity of each subject were ensured through coding of all data and protecting the obtained data.

\section{Procedure}

Data collection for this study was carried out through ten months starting from November 2019 to August 2020 (3 to 4 days per week) during morning or evening shift. Firstly, the investigator introduced her-self to the patients, explained the general purpose of the study \& asked them to give an oral consent for their participation.

The data was collected by the investigator in the first interview which took about 5-10 minutes from each client at the previous mentioned settings, after that the content of the educational brochure introduced for them concerning the educational protocol regarding post orthopedic surgery discharge instructions.

\section{Session plan}

Data collection for study sample was done in inpatient orthopedic department first during their hospitalization (ranged from 1to5 days) then in outpatient clinic for follow-up. The total number of sessions for data collection \& training session for the study group was 4-5 sessions. The duration of each session about $5-10$ minutes according to patient tolerance. At the end of each session discussion and feedback will be made.

At the end of the session, the researcher gave the study participants a handout (Photo brochure) as educational information and training practices for post orthopedic surgery discharge instructions, it was formulated by researcher after reviewing related literature. It was included an overview about deep venous thrombosis ,steps of application of elastic stocking, four types of exercise and four types of range of motion for strengthening thigh and calf muscles. Also, instructions regard duration and frequency to ensure that the patients perform these exercises accurately after their hospital discharge (4-6 weeks) and at home.

\section{Statistical analysis of data:}

Data were analyzed using the statistical package for social science (SPSS) version 20. Numerical data were expressed as means and SD. Quantitative data were expressed as frequency and percentage. For quantitative data; comparison between two variables were done using t-test, and comparison between more than two variables used ANOVA test paired t-test. Relations between different numerical variables were tested using Pearson correlation. Probability(Pvalue) less than 0.05 was considered significant and less than 0.01 was considered highly significant .

\section{Results}

Table 1 :Distribution of study and control groups regarding demographic and medical data $n=72$

\begin{tabular}{|c|c|c|c|c|c|c|c|}
\hline \multicolumn{2}{|r|}{ Items } & \multicolumn{2}{|c|}{$\begin{array}{c}\text { Study group } \\
\text { n }\end{array}$} & \multicolumn{2}{|c|}{$\begin{array}{c}\text { Control group } \\
\text { n }\end{array}$} & $\mathrm{X} 2$ & p. value \\
\hline \multicolumn{2}{|l|}{ Age by years } & \multicolumn{2}{|c|}{$43.63 \pm 12.49$} & \multicolumn{2}{|c|}{$39.94 \pm 10.29$} & 1.369 & .175 \\
\hline \multirow[t]{2}{*}{ Sex } & Male & 15 & 41.7 & 21 & 58.3 & \multirow{2}{*}{2.000} & \multirow{2}{*}{.119} \\
\hline & Female & 21 & 58.3 & 15 & 41.7 & & \\
\hline \multirow[t]{2}{*}{ Marital status } & Single & 7 & 19.4 & 5 & 13.9 & \multirow{2}{*}{.400} & \multirow{2}{*}{.377} \\
\hline & Married & 29 & 80.6 & 31 & 86.1 & & \\
\hline \multirow[t]{7}{*}{ Medical data } & Tibial fracture & 15 & 41.6 & $\mathbf{1 0}$ & 27.8 & \multirow{7}{*}{20.386} & \multirow{7}{*}{.118} \\
\hline & Femoral fracture & 5 & 13.8 & 3 & 8.3 & & \\
\hline & $\begin{array}{l}\text { Foot fractures (tarsals, } \\
\text { metatarsals and phalanges) }\end{array}$ & 2 & 5.6 & 4 & 11.2 & & \\
\hline & Ankle fracture & 4 & 11.2 & 5 & 13.8 & & \\
\hline & Hip joint dislocation & 2 & 5.6 & 4 & 11.2 & & \\
\hline & $\begin{array}{l}\text { Non united fracture shaft } \\
\text { tibia }\end{array}$ & 2 & 5.6 & 5 & 13.8 & & \\
\hline & Cruciate Ligament tear & 6 & 16.7 & 5 & 13.8 & & \\
\hline
\end{tabular}

Chi-Square Tests $\quad *=$ Significant difference $* \mathrm{p} \leq 0.05$

$* *=$ highly significance $* \mathrm{p} \leq 0.01 \quad \mathrm{Ns}=$ Non significant difference $\mathrm{P}>0.05$

Table 1: Shows that more than half $(58.3 \%)$ of study group were females, while more than half of patients in the control group $(58.3 \%)$ were males with mean age for the study and control groups (43.63 \pm 12.49 and $39.94 \pm 10.29$ years) respectively. The majority of both control and study groups were married. The highest percentage in both control and study group of patients had diagnosed with tibia fracture. 
Minia Scientific Nursing Journal (Print - ISSN 2537-012X) (Online - ISSN 2785-9797) Vol. (9) No. (1) June 2021

Table 2 : Comparison between study and control groups regarding risk factors $n=72$

\begin{tabular}{|c|c|c|c|c|c|c|}
\hline \multirow[t]{2}{*}{ Items } & \multicolumn{2}{|c|}{ Study group $=36$} & \multicolumn{2}{|c|}{ Control group $=36$} & \multirow[t]{2}{*}{$\mathrm{X} 2$} & \multirow[t]{2}{*}{ p.value } \\
\hline & $\mathbf{n}$ & $\%$ & $\mathbf{n}$ & $\%$ & & \\
\hline \multicolumn{7}{|l|}{ Age } \\
\hline 18-30yrs & 5 & 13.9 & 7 & 19.4 & \multirow{5}{*}{8.434} & \multirow{5}{*}{.077} \\
\hline 31-40yrs & 10 & 27.8 & 7 & 19.4 & & \\
\hline 41-50yrs & 9 & 25.0 & 18 & 50.0 & & \\
\hline 51-60yrs & 10 & 27.8 & 4 & 11.1 & & \\
\hline 61-65 yrs & 2 & $5.6 \%$ & 0 & 0.0 & & \\
\hline \multicolumn{7}{|l|}{ Mobility } \\
\hline Ambulant & 1 & 2.8 & 7 & 19.4 & \multirow{4}{*}{17.329} & \multirow{4}{*}{$.001 * *$} \\
\hline Limited & 10 & 27.8 & 21 & 58.3 & & \\
\hline Very limited & 20 & 55.6 & 7 & 19.4 & & \\
\hline Complete bed rest & 5 & 13.9 & 1 & 2.8 & & \\
\hline \multicolumn{7}{|l|}{ Trauma risk injury } \\
\hline Head injury & 1 & 2.8 & 0 & 0.0 & \multirow{3}{*}{2.057} & \multirow{3}{*}{.358} \\
\hline Pelvis injury & 1 & 2.8 & 0 & 0.0 & & \\
\hline Lower limb injury & 34 & 94.4 & 36 & 100.0 & & \\
\hline \multicolumn{7}{|l|}{ Body mass index } \\
\hline Average 20-25 & 14 & 38.9 & 10 & 27.8 & \multirow{4}{*}{6.158} & \multirow{4}{*}{.104} \\
\hline Over weight 26-30 & 14 & 38.9 & 8 & 22.2 & & \\
\hline Obese 31-40 & 7 & 19.4 & 16 & 44.4 & & \\
\hline Very obese $\geq 40$ & 1 & 2.8 & 2 & 5.6 & & \\
\hline \multicolumn{7}{|l|}{ Special risk factors } \\
\hline \multicolumn{7}{|l|}{ Oral contraceptive } \\
\hline 20-35yrs & 4 & 44.4 & 2 & 18.2 & \multirow{3}{*}{1.626} & \multirow{3}{*}{.217} \\
\hline More 35yrs & 5 & 55.6 & 9 & 81.8 & & \\
\hline Pregnancy & 8 & 100.0 & 2 & 100.0 & & \\
\hline \multicolumn{7}{|l|}{ High risk disease } \\
\hline Anemia( sickle anemia) & 4 & 11.1 & 5 & 13.9 & .127 & .500 \\
\hline Chronic heart disease & 10 & 27.8 & 0 & 0.0 & 11.613 & $.001 * *$ \\
\hline Varicose vein & 3 & 8.3 & 5 & 13.9 & .563 & .355 \\
\hline Cerebral vascular disease & 1 & 2.8 & 0 & 0.0 & 1.014 & .500 \\
\hline Pervious DVT & 1 & 2.8 & 0 & 0.0 & 1.014 & .500 \\
\hline \multicolumn{7}{|l|}{ Surgical intervention } \\
\hline Orthopedic surgery & 36 & 100.0 & 36 & 100.0 & $\begin{array}{ll}--- \\
\end{array}$ & $\begin{array}{ll}--- \\
\end{array}$ \\
\hline \multicolumn{7}{|l|}{ Risk category } \\
\hline High risk & 36 & 100.0 & 36 & 100.0 & $\begin{array}{ll}--- \\
\end{array}$ & $\begin{array}{ll}--- \\
\end{array}$ \\
\hline
\end{tabular}

Chi-Square Tests $\quad *=$ Significant difference $* \mathrm{p} \leq 0.05$

$* *=$ highly significance $* \mathrm{p} \leq 0.01 \quad \mathrm{Ns}=$ Non significant difference $\mathrm{P}>0.05$

Table 2: Regarding the risk factors of developing deep venous thrombosis among orthopedic surgical patients; the current study clarify that half of patients' age in the control group was ranging between $40-50$ years while more than quarter of the study group ranging between $30-40$ years and other quarter ranging between 50 -60 years old. Regarding the mobility; the present study shows that more than half of both study and control group experience a very limited and limited mobility respectively. Regarding the trauma risk injury; the majority of both two groups of patients $(94.4 \%$ and $100 \%)$ had lower limb injury. Regarding body mass index the present study revealed that more than one third in both study and control groups experience over weight and obesity respectively (38.9\% and 44.4\%). Regarding chronic diseases; More than one quarter in the study group (27.8\%) had chronic heart disease while the highest percentage in the control group had anemia (sickle cell anemia) and varicose vein. All patients (100\%) in both study and control groups were performing orthopedic surgeries with high risk category.

Table 3 : Deep venous thrombosis evaluation among study and control groups $n=72$

\begin{tabular}{|c|c|c|c|c|c|c|c|c|c|c|}
\hline \multirow[t]{2}{*}{ Signs and symptoms of DVT } & \multicolumn{4}{|c|}{ Study group $=36$} & \multicolumn{4}{|c|}{ Control group $=36$} & \multirow[t]{3}{*}{$\mathrm{X} 2$} & \multirow[t]{3}{*}{ p. value } \\
\hline & \multicolumn{2}{|c|}{ Yes } & \multicolumn{2}{|c|}{ No } & \multicolumn{2}{|c|}{ Yes } & \multicolumn{2}{|c|}{ No } & & \\
\hline Calf muscle & $\mathrm{n}$ & $\%$ & $\mathrm{n}$ & $\%$ & $\mathrm{n}$ & $\%$ & $\mathrm{n}$ & $\%$ & & \\
\hline - $\quad$ Pain & 8 & 22.2 & 28 & 77.8 & 6 & 16.7 & 30 & 83.3 & .355 & .383 \\
\hline tenderness & 3 & 8.3 & 33 & 91.7 & 7 & 19.4 & 29 & 80.6 & 1.858 & .154 \\
\hline Redness & 2 & 5.6 & 34 & 94.4 & 10 & 27.8 & 26 & 72.2 & 6.400 & $.012 *$ \\
\hline Warmth & 1 & 2.8 & 35 & 97.2 & 5 & 14.3 & 31 & 86.1 & 2.909 & .099 \\
\hline Swelling & 3 & 8.3 & 33 & 91.7 & 21 & 58.3 & 15 & 41.7 & 20.250 & $.001 * *$ \\
\hline Cramping & 1 & 2.8 & 35 & 97.2 & 5 & 14.3 & 31 & 86.1 & 2.909 & .099 \\
\hline Absent & 28 & 77.8 & 8 & 22.2 & 15 & 41.7 & 21 & 58.3 & 9.758 & $.002 * *$ \\
\hline \multicolumn{11}{|l|}{ Thigh muscle } \\
\hline - $\quad$ Pain & 7 & 19.4 & 29 & 80.6 & 3 & 8.3 & 33 & 91.7 & 1.858 & .154 \\
\hline - $\quad$ tenderness & 4 & 11.1 & 32 & 88.9 & 3 & 8.3 & 33 & 91.7 & .158 & .500 \\
\hline Redness & 1 & 2.8 & 35 & 97.2 & 3 & 8.3 & 33 & 91.7 & 1.059 & .307 \\
\hline - $\quad$ Warmth & 1 & 2.8 & 35 & 97.2 & 3 & 8.3 & 33 & 91.7 & 1.059 & .307 \\
\hline Swelling & 3 & 8.3 & 33 & 91.7 & 13 & 36.1 & 23 & 63.9 & 8.036 & $.005 * *$ \\
\hline - $\quad$ Cramping & 1 & 2.8 & 35 & 97.2 & 2 & 5.6 & 34 & 94.4 & .348 & .500 \\
\hline Absent & 29 & 80.6 & 7 & 19.4 & 23 & 63.9 & 13 & 36.1 & 2.492 & .094 \\
\hline
\end{tabular}


Table 3: demonstrates that there is a statistical significant difference between study and control group regarding signs and symptoms of DVT in calf muscle regarding redness, swelling and absence of signs with p.value $\left(0.012^{*}, 0.001 * *\right.$, and $\left.0.002^{* *}\right)$ respectively. While in thigh muscle presence of swelling show a statistical significant difference with p.value $\left(0.005^{* *}\right)$ between the two groups.

Table 4 : Comparison between study and control groups regading Doppler finding $\mathbf{n}=\mathbf{7 2}$

\begin{tabular}{|c|c|c|c|c|c|c|c|c|c|c|}
\hline \multirow[t]{3}{*}{ Doppler finding } & \multicolumn{4}{|c|}{ Study group $=36$} & \multicolumn{4}{|c|}{ Control group $=36$} & \multirow[t]{3}{*}{$\mathrm{X} 2$} & \multirow[t]{3}{*}{ p.value } \\
\hline & \multicolumn{2}{|c|}{ Present } & \multicolumn{2}{|c|}{ Absent } & \multicolumn{2}{|c|}{ Present } & \multicolumn{2}{|c|}{ Absent } & & \\
\hline & $\mathbf{n}$ & $\%$ & $\mathrm{n}$ & $\%$ & $\mathrm{n}$ & $\%$ & $\mathrm{n}$ & $\%$ & & \\
\hline Right limb DVT & 2 & 5.6 & 34 & 94.4 & 3 & 8.3 & 33 & 91.7 & .215 & .500 \\
\hline Left limb DVT & $\mathbf{0}$ & 0.0 & 36 & 100.0 & 5 & 13.9 & 31 & 86.1 & 5373 & $027 *$ \\
\hline
\end{tabular}

Table 4: Shows that there is a statistical significant difference between study and control groups regarding the occurrence of DVT especially Left limb DVT p.value $\left(0.027^{*}\right)$ after application of the nursing educational protocol.

\section{Discussion}

Deep Venous Thrombosis (DVT) is a potentially fatal complication (Pollack, 2011). It is responsible for most cases of venous thromboembolism Patients undergoing highrisk orthopedic procedures, specifically total hip arthroplasty, total knee arthroplasty, and hip fracture repair surgery are at a significantly increased risk without appropriate prophylaxis (Kamerkar, John, Desai, Dsilva, \& Joglekar, 2016).

Deep vein thrombosis (DVT) is a serious condition that occurs when a blood clot forms in a vein located deep in the body. A blood clot is a clump of blood that's turned to a solid state. Deep vein blood clots typically form in the thigh or lower leg, but they can also develop in other areas of the body. Other names associated with this condition may include thromboembolism, post-thrombotic syndrome, and postphlebitic syndrome (Delgado, 2019).

The aim of this study was to evaluate the effect of implementing nursing educational protocol on reducing of deep vein thrombosis among orthopedic patients. The study hypothesed that the occurrence of deep venous thrombosis will be reduced in orthopedic surgery patients who are following nursing education protocol.

The present study demonstrates that more than half of study group are females, while more than half of patients in the control group were males with mean age for the study and control groups $(43.63 \pm 12.49$ and $39.94 \pm 10.29$ years $)$ respectively. (Mohamed, Othman, El Alphy, \& Sheble, 2017) were disagreeing with the current study results as they reported that "more than half of the participants aged from 50 to 60 years with mean age 47.3. The highest proportions of study participants were female.

The current study shows that the highest percentage in both control and study group of patients had diagnosed with tibia fracture.(Hussein, Mohammad, Elkady, \& Ghanem, 2020) were disagreeing with current study results as they revealed that" In relation to surgically treated joint, right hip was the commonest. Finally, regarding the reason for surgical replacement, around two-third of patients suffered from osteoarthritis".

The patients assigned to the study and control groups of the present study were selected to have similar sociodemographic. This is of major importance since variables such as patient age, gender, as well as body weight may influence the risk of occurrence of DVT.

Regarding the risk factors of developing deep venous thrombosis among orthopedic surgical patients; the current study clarify that half of patients' age in the control group was ranging between forty and fifty years while more than quarter of the study group ranging between thirty and forty years and other quarter ranging between fifty and sixty years old. (Anderson et al., 2019) were in the same line as they revealed that" the present study results reported that the risk of development of DVT had a significant association with patient's age, with the risk increasing with older age. In line with this, a study in Turkey demonstrated that patients' age was a significant factor that increased the DVT risk among orthopedic surgery patients".

Regarding the mobility; the present study shows that more than half of both study and control group experience a very limited and limited mobility respectively. (Gould et al., 2012) were supporting the study results as they reported that "In surgical patients, the risk of DVT associated with immobilization is well recognized. Postoperative immobility is an inherent consequence of the surgery itself and, therefore, it is difficult to separate the relative contributions of surgery and immobility as they are not strictly independent variables".

Regarding the trauma risk injury; the majority of both two groups of patients had lower limb injury. (Chu \& Haga, 2015) who perform a study titled" Venous thromboembolism associated with lower limb fractures after trauma: dilemma and management" were in the same line as they revealed that "Trauma patients have the highest risk of developing venous thromboembolism (VTE) among hospitalized patients, with a reported thirteen-fold greater risk of developing VTE over non-trauma patients. Major lower limb trauma patients have an approximate six-fold increased risk of developing VTE during admission compared with minor trauma patients".

Regarding body mass index the present study revealed that more than one third in both study and control groups experience over weight and obesity respectively. (Lorenzet, Napoleone, Cutrone, \& Donati, 2012) were agreeing with the study results as they reported that" Obesity is associated with inactivity, raised intraabdominal pressure and decreased blood velocity in the legs, as well as with pro inflammatory and prothrombotic states. These effects are all likely to contribute to the risk of venous thromboembolism; that is, deep venous thrombosis (DVT) with or without complication by pulmonary embolism (PE). Obesity is associated with almost doubling the risk of primary VTE".

Furthermore,(Lorenzet et al., 2012) and (Klovaite, Benn, \& Nordestgaard, 2015) found that "associations of BMI and waist circumference were somewhat stronger with PE vs. DVT and about twice as strong with VTE vs. CHD. These data suggest that efforts to combat the entire spectrum of obesity and overweight should yield important benefits for VTE prevention".

Regarding chronic diseases; More than one quarter in 
the study group had chronic heart disease while the highest percentage in the control group had anemia (sickle cell anemia) and varicose vein.(Chi et al., 2018)who performed a study titled" Association of Anemia with Venous Thromboembolism in Acutely Ill Hospitalized Patients" were agreeing with study findings as they revealed that" decreased hemoglobin concentration (anemia) was associated with a two-fold risk of symptomatic DVT or non-fatal PE, despite the provision of pharmacologic thromboprophylaxis with enoxaparin or betrixaban, an FDA approved direct oral anticoagulant for VTE prevention".

Also, (Tang, Wu, Lip, Yin, \& Hu, 2016)who performed a study titled "Heart failure and risk of venous thromboembolism: a systematic review and meta-analysis" enforce the current study results as they reported that" Reduced heart function with symptoms of congested lungs, fluid and water retention, rapid or irregular heartbeats as a result of congestive heart failure has recently been determined to be an independent risk factor for VTE Additionally, according to a meta-analysis, hospitalized patients with heart failure had an increased risk for VTE in Asians".

The current study results revealed that there was a statistical significant difference between study and control group regarding signs and symptoms of DVT in calf muscle regarding redness, swelling and absence of signs while in thigh muscle presence of swelling show a statistical significant difference between the two groups.

(Patel, Chun, \& Brenner, 2019) were in the same line with the current study results as they reported that" Deep venous thrombosis (DVT) classically produces pain and limb edema; however, in a given patient, symptoms may be present or absent, unilateral or bilateral, or mild or severe. Thrombus that does not cause a net venous outflow obstruction is often asymptomatic. Edema and swelling is the most specific symptom of DVT".

There was a statistical significant difference between study and control groups regarding the occurrence of DVT after application of the nursing educational protocol. From the researcher point of view these results are accepted as the researchers provided a valuable nursing educational protocol to study group of patients which has the clear effect on reducing the number of patients who developed DVT than the control group. Also, (Mohamed et al., 2017) who performed a study titled" Effect of Implementing Nursing Care Guidelines on the Occurrence of Deep Vein Thrombosis among Orthopedic Patients" were in the same line with study results as they revealed that" The intervention is also beneficial in terms of patient outcomes, with significantly lower DVT risk grade and score compared with the control group patients".

Additionally,(Yin \& Shan, 2015) clarify that" There was a statistically significant difference between DVT incidence in the two groups, where the number of patients with DVT in the intervention group was lower due to the distinct risk levels and corresponding nursing intervention".

\section{Conclusions:}

Application of educational protocol was effective in reducing DVT among orthopedic surgical patients.

\section{Recommendations:}

Recommendations for Orthopedic department and nurses:

- Orthopedic surgical patient's risk for developing deep vein thrombosis should be assessed by nurses constantly and progressively.

- A continuous educational and training program for staff nurses planned and offered on regular basis for patients undergoing orthopedic surgery, including post orthopedic surgery discharge instructions and home care.

- Written Arabic booklets or brochure and posters including post orthopedic surgery discharge instructions should be available at health care settings and given to orthopedic surgical patients and their care givers.

- The mass media should be used more effectively to improve awareness of the public specially the older adults and their care giver about disease, methods of treatment and prevention.

- Long-term follow-up of Orthopedic surgical patient's is necessary to determine long-term outcomes.

\section{Recommendations for Patients:}

Planning educational classes for orthopedic surgical patients about risk factors, treatment, complications and prevention of DVT following orthopedic surgery, through home visit in their health care settings.

\section{Recommendations for further researches:}

Replication of the current study on a larger probability sample from different geographical areas to achieve generalizable results.

\section{References}

(1) Akpinar, E. E., Hoşgün, D., Akan, B., Ateş, C., \& Gülhan, M. (2013). Does thromboprophylaxis prevent venous thromboembolism after major orthopedic surgery? Jornal brasileiro de pneumologia : publicacao oficial da Sociedade Brasileira de Pneumologia e Tisilogia, 39(3), 280-286. doi: 10.1590/S1806-37132013000300004

(2) Anderson, D. R., Morgano, G. P., Bennett, C., Dentali, F., Francis, C. W., Garcia, D. A., . . . Dahm, P. (2019). American Society of Hematology 2019 guidelines for management of venous thromboembolism: prevention of venous thromboembolism in surgical hospitalized patients. Blood Advances, 3(23), 3898-3944.

(3) Attia, A., Ali, H., Al Amory, M., \& Othman, H. (2015). Thromboprophylaxis does not prevent venous thromboembolism after major surgery. Egyptian Journal of Chest Diseases and Tuberculosis, 64(1), 249-254. doi: https://doi.org/10.1016/j.ejcdt.2014.11.011

(4) Autar, R. (2003). The management of deep vein thrombosis: the Autar DVT risk assessment scale re-visited. Journal of Orthopaedic Nursing, 7(3), 114-124. doi: 10.1016/s13613111(03)00051-7

(5) Chi, G., Gibson, C. M., Hernandez, A. F., Hull, R. D., Kazmi, S. H. A., Younes, A., . . . Goldhaber, S. Z. (2018). Association of Anemia with Venous Thromboembolism in Acutely Ill Hospitalized Patients: An APEX Trial Substudy. AJM The American Journal of Medicine, 131(8), 972.e971-972.e977.

(6) Chu, C. C., \& Haga, H. (2015). Venous thromboembolism associated with lower limb fractures after trauma: dilemma and management. JOURNAL OF ORTHOPAEDIC SCIENCE, 20(2), 364-372.

(7) D'Alesandro, M. (2016). Focusing on lower extremity DVT. Nursing, 46(4), 28-35; quiz 35-26. doi: 10.1097/01.nurse.0000481417.73469.00

(8) Dahl, B. I. E. a. O. E. ( 2013 ). Thromboembolism in Orthopedic Surgery: Scope of the Problem Thromboembolism in Orthopedic Surgery (pp. 1). London Heidelberg New York Dordrecht Springer.

(9) Delgado, A. (2019). Everything You Want to Know About Deep Vein Thrombosis (DVT). health line. 
(10) Flevas, D. A. Megaloikonomos, P. D., Dimopoulos, L. Mitsiokapa, E., Koulouvaris, P., \& Mavrogenis, A. F. (2018). Thromboembolism prophylaxis in orthopaedics: an update. EFORT open reviews, 3(4), 136-148. doi: 10.1302/20585241.3.170018

(11) Francis, C. W. (2013). Prevention of VTE in patients having major orthopedic surgery. J Thromb Thrombolysis, 35(3), 359-367. doi: 10.1007/s11239-013-0889-9

(12) Gould, M. K., Garcia, D. A., Wren, S. M., Karanicolas, P. J., Arcelus, J. I., Heit, J. A., \& Samama, C. M. (2012). Prevention of VTE in nonorthopedic surgical patients. Antithrombotic therapy and prevention of thrombosis, 9th ed: American College of Chest Physicians evidence-based clinical practice guidelines. Chest Chest, 141(2 SUPPL.), e227S-e277S

(13) Hussein, A., Mohammad, Z., Elkady, H., \& Ghanem, H. (2020). Effect of Educational Nursing Protocol on Minimizing Venous Thromboembolism for Patients Undergoing Simultaneously Arthroscopic ACLR and High Tibial Osteotomy. Assiut Scientific Nursing Journal Assiut Scientific Nursing Journal, 8(20), 75-84.

(14) Kamerkar, D. R., John, M. J., Desai, S. C., Dsilva, L. C., \& Joglekar, S. J. (2016). Arrive: A retrospective registry of Indian patients with venous thromboembolism. Indian Journal of Critical Care Medicine : Peer-reviewed, Official Publication of Indian Society of Critical Care Medicine, 20(3), 150-158.

(15) Klovaite, J., Benn, M., \& Nordestgaard, B. G. (2015). Obesity as a causal risk factor for deep venous thrombosis: a Mendelian randomization study. J Intern Med Journal of Internal Medicine, 277(5), 573-584.

(16) Lorenzet, R., Napoleone, E., Cutrone, A., \& Donati, M. B. (2012). Thrombosis and obesity: Cellular bases. TR Thrombosis Research, 129(3), 285-289.

(17) Mohamed, A. A. H., Othman, W. N., El Alphy, B. S., \& Sheble, A. M. (2017). Effect of Implementing Nursing Care Guidelines on the Occurrence of Deep Vein Thrombosis among Orthopedic
Patients. IOSR JNHS IOSR Journal of Nursing and Health Science, 06(03), 28-36.

(18) Naing, L., Winn, T., \& Rusli Bin, N. (2006). Practical issues in calculating the sample size for prevalence studies.

(19) Patel, K. K., Chun, L. J., \& Brenner, B. E. (2019). What are the signs and symptoms of deep venous thrombosis (DVT)?

(20) Phillippe, H. M. (2017). Overview of venous thromboembolism. The American journal of managed care, 23(20), 376.

(21) Pollack, C. V., Jr. (2011). Advanced management of acute iliofemoral deep venous thrombosis: emergency department and beyond. Annals of emergency medicine, 57(6), 590-599.

(22) Rahman, W. A., Habsa, G. H., Hammad, M., Selim, N. M., Hammad, A., \& Al-Mohrej, O. A. (2020). Incidence of silent venous thromboembolism after total hip arthroplasty: A comparison of rivaroxaban and enoxaparin. J. Orthop. Surg. Journal of Orthopaedic Surgery, 28(2).

(23) Schellack, G., Modau, T., \& Schellack, N. (2016). Clinical overview of venous thromboembolism. SA pharmaceutical journal, 58(1), 39-45.

(24) Tang, L., Wu, Y.-Y., Lip, G. Y. H., Yin, P., \& Hu, Y. (2016) Heart failure and risk of venous thromboembolism: a systematic review and meta-analysis. LANHAE The Lancet Haematology, 3(1), e30-e44.

(25) Thompson, A. E. (2015). Deep vein thrombosis. : Centers for Disease Control and Prevention National Heart, Lung, and Blood Institute, 311(7), 717-728.

(26) Xu, Y., Zhao, J., \& Chen, Y. (2018). Prevention of venous thromboembolism in patients undergoing major orthopedic surgery in China: a qualitative study of patients' perceptions. J Orthop Surg Res, 13(1), 98-98. doi: 10.1186/s13018-018-0813-2

(27) Yin, H.-Z., \& Shan, C.-M. (2015). The effect of nursing intervention based on Autar scale results to reduce deep venous thrombosis incidence in orthopaedic surgery patients. International Journal of Nursing Sciences, 2(2), 178-183. doi: 10.1016/j.ijnss.2015.04.003 